CHOLERA

\section{Integrated view of Vibrio cholerae in the Americas}

\author{
Daryl Domman, ${ }^{1 *}$ Marie-Laure Quilici, ${ }^{2}$ Matthew J. Dorman, ${ }^{1}$ Elisabeth Njamkepo, ${ }^{2}$ \\ Ankur Mutreja, ${ }^{1,3}$ Alison E. Mather,${ }^{1,4}$ Gabriella Delgado, ${ }^{5}$ Rosario Morales-Espinosa, ${ }^{5}$ \\ Patrick A. D. Grimont, ${ }^{6}$ Marcial Leonardo Lizárraga-Partida, ${ }^{7}$ Christiane Bouchier, ${ }^{8}$ \\ David M. Aanensen, ${ }^{9}$ Pablo Kuri-Morales, ${ }^{10}$ Cheryl L. Tarr, ${ }^{11}$ Gordon Dougan, ${ }^{1,3}$ \\ Julian Parkhill, ${ }^{1}$ Josefina Campos, ${ }^{12}$ Alejandro Cravioto, ${ }^{13}$ \\ François-Xavier Weill, ${ }^{1,2} \dagger$ Nicholas R. Thomson ${ }^{1,14 *} \dagger$
}

Latin America has experienced two of the largest cholera epidemics in modern history; one in 1991 and the other in 2010. However, confusion still surrounds the relationships between globally circulating pandemic Vibrio cholerae clones and local bacterial populations. We used whole-genome sequencing to characterize cholera across the Americas over a 40-year time span. We found that both epidemics were the result of intercontinental introductions of seventh pandemic El Tor $V$. cholerae and that at least seven lineages local to the Americas are associated with disease that differs epidemiologically from epidemic cholera. Our results consolidate historical accounts of pandemic cholera with data to show the importance of local lineages, presenting an integrated view of cholera that is important to the design of future disease control strategies.

C holera is an acute intestinal infection that leads to a rapid and severe dehydrating diarrhea, and is caused by serogroup $\mathrm{O1}$ and 0139 Vibrio cholerae. The global disease burden of cholera is estimated to be between 1.3 and 4 million cases a year with 21,000 to 143,000 deaths (I). The current seventh pandemic (7P) of cholera began in 1961 and is attributed to a $V$. cholerae $\mathrm{O} 1$ biotype $\mathrm{El}$ Tor lineage, which is different from the Classical biotype V. cholerae $\mathrm{O} 1$ thought to be responsible for previous pandemics. Aside from being a prominent human pathogen, exploratory analyses have demonstrated since the 1970 s that $V$. cholerae is an integral member of many coastal, estuarine, and brackish water ecosystems, as are other Vibrio species, in which it is often associated with copepods and zooplankton (2). Accordingly, a view of $V$. cholerae

\footnotetext{
Wellcome Trust Sanger Institute, Wellcome Genome Campus, Hinxton CB10 1SA, UK. 'Institut Pasteur, Unité des Bactéries Pathogènes Entériques, Paris, 75015, France. ${ }^{3}$ Department of Medicine, University of Cambridge, Addenbrooke's Hospital, Cambridge CB2 OSP, UK. ${ }^{4}$ Department of Veterinary Medicine, University of Cambridge, Madingley Road, Cambridge, CB3 OES, UK. ${ }^{5}$ Department of Microbiology and Parasitology, Faculty of Medicine, Universidad Nacional Autónoma de México, Mexico, D.F., Mexico. ${ }^{6}$ Institut Pasteur, Unité Biodiversité des Bactéries Pathogènes Emergentes, Paris, 75015, France. ${ }^{7}$ Centro de Investigación Científica y de Educación Superior de Ensenada, Baja California, (CICESE), Ensenada, Baja California, Mexico. ${ }^{8}$ Institut Pasteur, Plate-forme Génomique (PF1), Paris, 75015, France. ${ }^{9}$ Centre for Genomic Pathogen Surveillance, Wellcome Genome Campus, Hinxton, Cambridge CB10 1SA, UK. ${ }^{10}$ Subsecretaría de Prevención y Promoción de la Salud, Secretaría de Salud, Ciudad de México, Mexico. ${ }^{11}$ Enteric Diseases Laboratory Branch, Centers for Disease Control and Prevention, Atlanta, GA, USA. ${ }^{12}$ Instituto Nacional de Enfermedades Infecciosas, ANLIS, Buenos Aires, Argentina. ${ }^{13}$ Faculty of Medicine, Universidad Nacional Autónoma de México, Mexico, D.F., Mexico. ${ }^{14}$ London School of Hygiene and Tropical Medicine, London WC1E 7HT, UK. *Corresponding author. Email: dd6@sanger.ac.uk (D.D.); nrt@sanger. ac.uk (N.R.T.) †These authors contributed equally to this work.
}

epidemiology emerged in the following decades, which posits that locally evolving, but globally distributed, V. cholerae populations are responsible for cholera outbreaks, which occur when climatic or environmental stimuli provide favorable bacterial growth conditions in these environs $(3,4)$. This perception has had profound effects on all levels of global public health; cholera is now considered to be ineradicable because its etiological agent is ubiquitous in aquatic ecosystems $(3,5)$.

Despite advances in our understanding of the global epidemiology of cholera, we still face unanswered fundamental questions about the relationships between local and global $V$. cholerae populations. Latin America presents a notable opportunity to investigate these relationships. Although this region has local foci of endemic $V$. cholerae, such as on the Gulf Coast of the United States and Mexico (6), pandemic cholera was absent from Latin America for nearly 100 years. In January 1991, a cholera outbreak occurred along the coast of Peru and spread rapidly to nearly every country in Latin America, causing 1.2 million disease cases and 12,000 deaths by 1997 (7). More recently, pandemic cholera was introduced into Haiti (8), where the resultant epidemic has affected more than 797,000 people and caused over 9400 deaths (9). In response to these two large-scale epidemics, regional and national surveillance systems in Latin America were hyperalert for cholera outbreaks, and as a result, their sampling framework captured a diverse collection of $V$. cholerae during both epidemic and interepidemic periods $(10,11)$. Coupling these precise epidemiological data, which describe the beginning of the epidemic, to increased sampling within Latin America, allows studies within this region to offer unprecedented opportunities to address the relationships be- tween local populations and globally circulating pandemic lineages of $V$. cholerae.

The relationships between these bacterial populations have been difficult to characterize until now, primarily due to the molecular methods used to assess the relatedness of $V$. cholerae isolates to one another $(10,12-14)$. Inconsistency in applying and interpreting results generated using these methods, such as the use of different restriction enzymes for pulsed-field gel electrophoresis and ribotyping, and the lack of standardized nomenclatures, has further complicated comparisons between studies. However, it is possible now to unify these results by using wholegenome sequencing.

To examine the relationship between $V$. cholerae lineages in Latin America, we sequenced a collection of 252 isolates. Phylogenetic analysis showed that 164 strains were of the $7 \mathrm{P} \mathrm{El} \mathrm{Tor} \mathrm{(7PET)}$ cholera lineage and 88 were strains distinct from 7PET (collectively referred to as non-7PET) (15). This collection is geographically and temporally broad, and includes representative isolates from 14. countries spanning 1974 to 2014, including preepidemic, epidemic (isolated and typed during the 1991 and Haitian epidemics), and interepidemic periods. Critically, this collection includes serogroup $\mathrm{O} 1$ and non-O1 isolates, both clinical and environmental isolates (figs. S1 to S3 tables S1 to $S 3$, and supplementary text note 1), isolates typed by early molecular approaches (table S4) $(11,12)$, and several key additional 7PET isolates from Africa [see companion analysis of Weill et al. (16)].

We placed these isolates into a phylogenetic framework, and determined the evolutionary relationships between lineages in Latin America. A global phylogeny, comprising a total of 665 isolates, revealed a marked diversity of $V$. cholerae lineages present in this region (Fig. 1 and figs. S2 and S3). Representative isolates from both the 1991 and 2010 epidemics clustered within the 7PET lineage (table S4). The phylogeny also revealed that isolates sampled in different years, and in some cases across multiple countries, comprise 11 lineages in Latin America (Fig. 1 and fig. S4). These lineages include Classical V. cholerae isolated from Mexico during the mid-1990s, as well as several $V$. cholerae $\mathrm{O} 1$ local lineages, such as the Gulf Coast lineage (17), or those containing isolates of Mx1 to Mx3 ribotypes (MX-1 to MX-3 lineages) described in Mexico (11). The Tucumán variant from Argentina (18) and the Amazonia variant from Brazil (19) form a single lineage, named Endemic Latin American 1 (ELA-1), in which these isolates remain clearly separated phylogenetically by their country of origin (Fig. 1A and fig. S2). Although 7 of the 18 samples sequenced from the Tucumán and other regions of Argentina belong to ELA-1, the other samples are distributed among five other lineages. More than 30 additional isolates sampled across Latin America do not belong to any previously known lineage and comprise at least eight different serotypes (fig. S5 and table S2).

Local V. cholerae O1 lineages in Latin America harbor a wide range of genetic determinants that are associated with pandemic disease (figs. S6 and 
S7 and tables S2 and S3). For instance, the genes encoding the bipartite cholera toxin (ctx $A B$ ), the primary virulence determinant of cholera borne by the lysogenic $\operatorname{CTX} \varphi$ bacteriophage, are present in several isolates from the Gulf Coast lineage and the MX-2 lineage (fig. S7 and tables S2 and S3). Typing based on the $c t x B$ locus revealed that these lineages harbor different variants of the cholera toxin. Several isolates unaffiliated with lineages sampled from Argentina and the U.S./Mexican Gulf Coast also harbor ctxAB. Notably, the genes encoding the toxin co-regulated pilus (TCP), which allows $V$. cholerae to colonize the human intestine successfully, are present in all ELA-3, Gulf Coast, and MX-2 isolates, whether or not they are $\mathrm{CTX} \varphi^{+}$ (fig. S7 and tables S2 and S3). The presence of TCP (if expressed) confers upon CTX $\varphi$-negative strains the potential to be infected and lysogenized by CTX $\varphi$ to become cholera toxin producers.

Our results support previous descriptions of isolates from the Classical lineage in Mexico between 1995 and $1997(20,21)$. This finding is notable because it was thought that this biotype had disappeared, globally, in the late 1980s (20). A recent study in Thailand, however, recovered Classical strains isolated as late as 2000 (22), indicating that this lineage persisted longer than previously thought. It has been proposed that Classical cholera isolates were present in Mexico more than a decade earlier and persisted there until the mid-1990s (21) because of a single case, imported into the United States from Cancún, Mexico, in 1983 (23, 24). This isolate was subsequently shown to be a member of the Gulf Coast

A

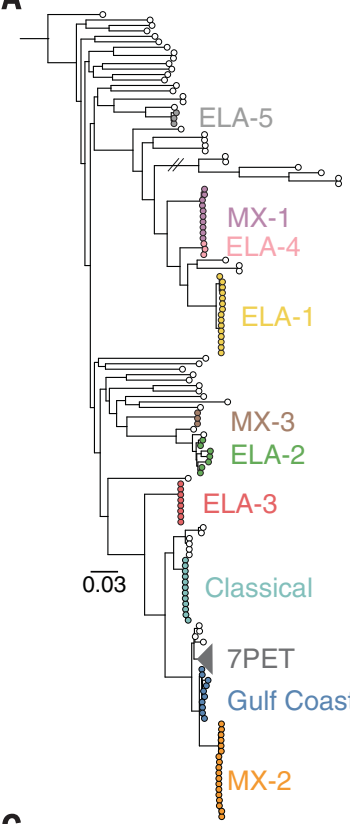

B

C

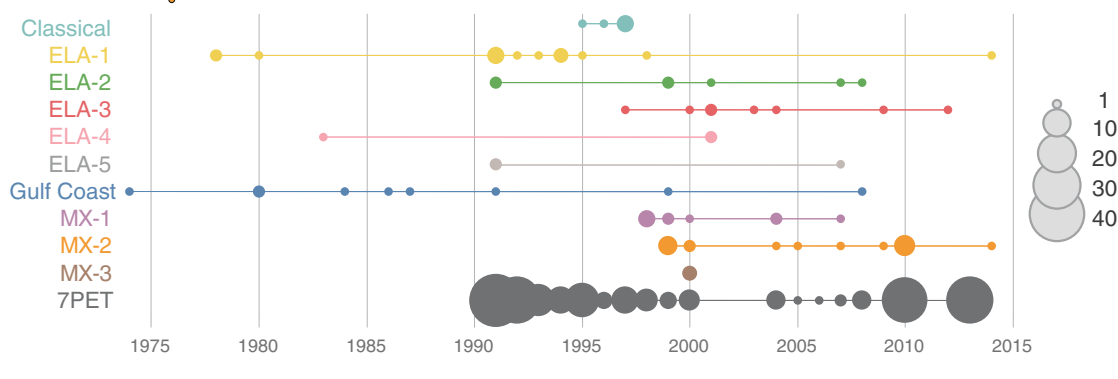

Year of isolation

Fig. 1. Multiple lineages of $\boldsymbol{V}$. cholerae are present in Latin America. (A) Maximum likelihood phylogeny of $148 \mathrm{~V}$. cholerae genomes. Local lineages present in Latin America are highlighted. The 7PET lineage is shown as a collapsed triangle. Four 7PET genomes

(reference genome N16961, and examples of LAT-1 to -3) were used as representatives of the 518 7PET genomes in this study. The scale bar denotes substitutions per variable site. The hash mark denotes a branch that was artificially shortened; the full tree is shown in figs. S2 and S3.

(B) Geographical distribution of selected local V. cholerae lineages in Latin America. The size of the circle denotes the number of genomes analyzed from that area. Only isolates annotated with explicit geographic information are shown. (C) Temporal distribution of genomes sampled from V. cholerae lineages present in Latin America. The size of the circle scales with the number of genomes in our study for each lineage. lineage by ribotyping and electrophoretic typing (ET) (23-25). Our phylogenetic data show that Classical biotype isolates in Mexico in the mid1990s are part of the Classical lineage, and not derived from the Gulf Coast lineage (Fig. 1 and fig. S2).

To understand better the relationships within the 7PET lineage, we calculated a robust maximum likelihood phylogeny from 518 7PET genomes. We detected a strong temporal signal, which allowed us to estimate dated phylogenies (Fig. 2 and fig. S8). These data show that the Latin American cholera epidemics were the result of multiple intercontinental introductions (Fig. 2), which we refer to as LAT (Latin American transmission) 1 to 3 . Our phylogeny reveals that two independent intercontinental introductions of 7PET V. cholerae into Latin America contributed to the 1991 epidemic (Fig. 2). The major epidemic clone during the 1991 epidemic is represented by the LAT-1 sublineage, corresponding phylogenetically to the previously described 7PET pandemic wave 1 isolates that form the West-African South American (WASA) lineage (26); these were originally typed as serotype Inaba, ribotype 5, and ET 4 (table S4) (12). We identified a frame-shift mutation at position 165 in the wbeT gene, consistent with the Inaba serotype in this lineage (table S1). LAT-1 isolates carry the El Tor variant of $\operatorname{ctx} B$ (ctxB3). The direct ancestors of LAT-1 in our phylogeny are isolates from Western and Central Africa (Angola, Cote d'Ivoire, Sao Tome) from the late 1980s. These African isolate genomes and the LAT-1 sublineage are separated by only 13 nonrecombinant single-nucleotide polymorphisms (SNPs). Further support for an African ancestry is the placement of African isolates (Uganda 1992, Nigeria 1997) within the LAT-1 sublineage. Several lines of evidence suggest that this introduction occurred close in time to the recorded start of the epidemic. First, our time-resolved phylogenies date this introduction to between 1985 and 1989, and the most recent common ancestor of LAT-1 isolates to 1989 (Fig. 2A). Second, our data show that genetic features that define the LAT-1 sublineage-the VSP-II gene variants (insertion between VC_0510 and VC_0516) and the WASA-1 genomic island-were acquired successively and in Africa during the late 1980s prior to this introduction (Fig. 2A and fig. S9). Moreover, Western Africa experienced cholera outbreaks immediately before the Peruvian epidemic (fig. S10). Tests for vibriocidal antibodies in stored sera from Lima, Peru, in 1990 indicate that $V$. cholerae was not present at this time (25). LAT-1 isolates were isolated in Mexico until 2010 (Fig. 2C). The more recent LAT-1 isolates collected between 2004 and 2010 in Mexico harbored a truncated $\operatorname{CTX} \varphi$ duplication and represent localized adaptations of these 7PET strains (27) (Fig. 2A).

The second clone introduced into Latin America in 1991 was described as serotype Ogawa, ribotype 6a, ET3 (12, 13), and resistant to furazolidone, sulfisoxazole, and streptomycin (13). This clone was first detected in a mountainous village near Mexico City, Mexico, in June 1991 and is believed to have been imported via coca smugglers using 
A

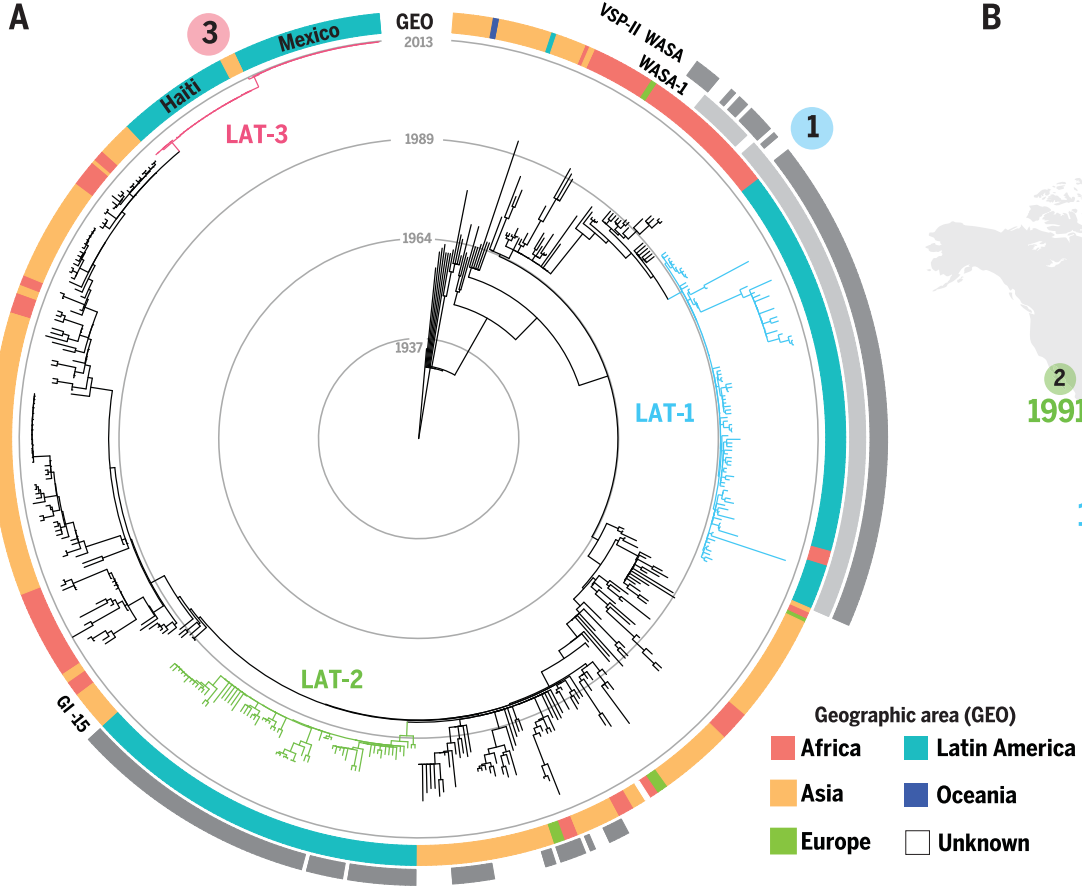

2

C

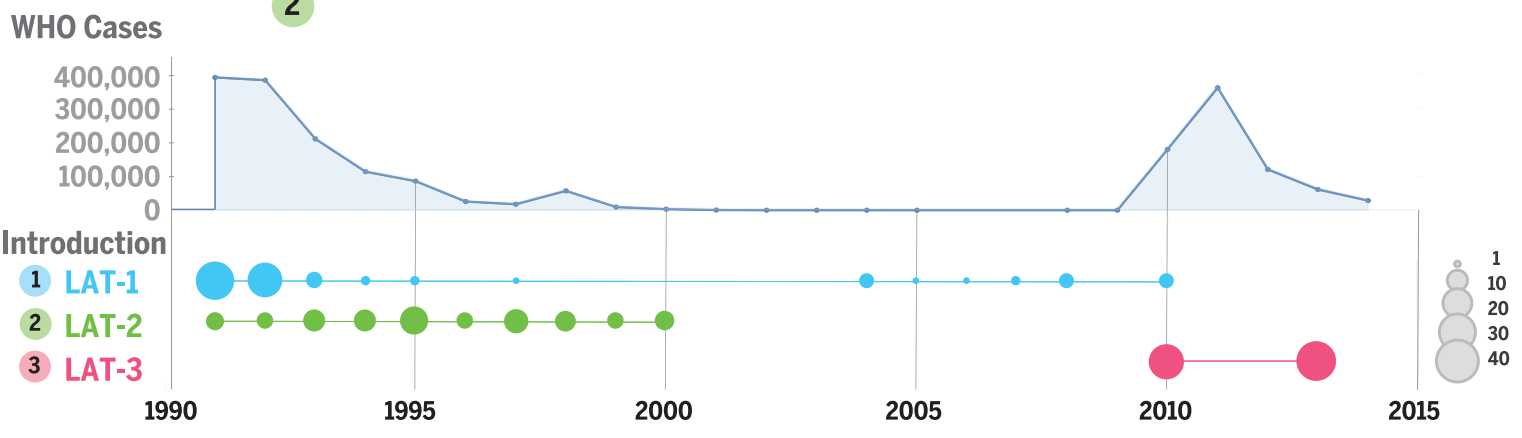

Fig. 2. Intercontinental introductions of seventh pandemic $V$. cholerae El Tor into Latin America. (A) Time-scaled maximum likelihood phylogeny of 518 7PET genomes. Inner rings denote time in years. Colored branches correspond to sublineages introduced into Latin America and are labeled LAT-1 to -3 . The geographic location of the isolates corresponds to the colored block in the GEO legend. Key genomic features that define lineages are shown in gray in outer bands. The introduction events are numbered on the outside of the circle. (B) Intercontinental introductions of seventh pandemic cholera into Latin America. Introductions are indicated by solid lines. The direct introduction of the LAT-2 sublineage from South Asia or China or introduction via Eastern Europe is uncertain and is denoted by dashed lines. The year of the first appearance of these lineages in Latin America is indicated. (C) Temporal distribution of the number of cases reported to the World Health Organization (WHO) in Latin America and number of genomes sampled in this study per LAT sublineage. The size of the circle scales with the number of genomes in our study for each sublineage. nearby private airstrips (28). This clone subsequently spread throughout Central America (13). After 1993, this was the major clone circulating in Mexico, where it persisted until 2000 (Fig. 2C) (11). Our phylogeny shows that the introduction of this second sublineage (LAT-2) occurred between 1987 and 1989, making this event concurrent with that of the LAT-1 introduction (Fig. 2A). The distinctive drug resistance profile of the LAT-2 sublineage was linked to the presence of a genomic island (GI-15) (Fig. 2A and figs. S9 and S11). GI-15 harbors the genes responsible for streptomycin $(a a d A)$ and sulfisoxazole (sull) resistance. With the exception of a few sporadic isolates, LAT-1 isolates were pan-susceptible to antimicrobials, and all lacked GI-15. The most closely related isolates to those of LAT-2 are those that were collected in South and Southeast Asia, Western Asia (Lebanon), and Eastern Europe (Romania) (Fig. 2A and table S1), many of which also harbor GI-15. These globally circulating wave 2 isolates, including the LAT-2 sublineage, also harbor $c t x B 1$ (fig. S9) with CTX $\varphi$ integrated into the smaller chromosome. Thus, the previously identified 7PET lineage harboring ctxB1 in Mexico (20) was not a local lineage, but was derived from the LAT-2 introduction. Our phylogeny indicates that this lineage originated from South or Southeast Asia (Fig. 2), from where it radiated globally. However, we cannot rule out that the introduction into Mexico came via secondary site(s) and not directly from Asia (Fig. 2, A and B).
The third introduction (LAT-3) involved the import of a South Asian strain into Haiti in 2010 and has been well documented $(8,29,30)$. The Haitian clone has been imported into surrounding countries, including Cuba, the Dominican Republic, the United States, and Mexico (31,32) (Fig. 2). An outbreak in 2013 within the Mexican region of Hidalgo was suspected to be the result of an import of the Haitian clone $(32,33)$. Our phylogeny indicates that these isolates descended from the Haitian (LAT-3) sublineage (Fig. 2) and share key genomic features, including the $c t x B 7$ variant and a characteristic deletion within VSP-II ( $\left.\triangle V C \_0495-V C \_0512\right)(29)$ (fig. S9).

Combined, we observe that $V$. cholerae lineages are associated with three distinct patterns of 
diarrheal disease within Latin America. First, there are lineages responsible for sporadic cases or limited outbreaks, in which secondary infections are rare or nonexistent (Fig. 1C) (11). Second, lineages that occupy long-term environmental reservoirs (such as the Gulf Coast lineage) cause illness over longer periods of time and across larger geographic areas (Fig. 1C and fig. S4). The third pattern, caused by pandemic V. cholerae, is visibly distinct. Pandemic lineages are responsible for massive, explosive epidemics that occur over short periods of time. The epidemiological distinction between local and pandemic lineages is starknearly 20,000 cases per week were seen at the beginning of the 1991 epidemic in Peru (28), and more than 250,000 cases were seen over 6 months at the beginning of the 2010 Haitian epidemic. By contrast, only 65 infections reported over a 20 -year period in the USA were associated with the Gulf Coast reservoir (34). We expand upon these definitions in supplementary text note 2 .

We show conclusively that both historical cholera epidemics within Latin America were the result of intercontinental introductions of globally circulating 7PET lineages and were not derived from indigenous local lineages. These data (i.e., the introduction of LAT-1 from Africa) also do not support the hypothesis that El Niño was responsible for the introduction of cholera in Peru in 1991 by potentiating the long-distance transport of aquatic pathogens from Asia through a biological corridor (35) or due to a surge in preexisting local lineages $(36,37)$ (Figs. land 2). Our data are instead consistent with descriptions of how cholera was introduced into Haiti in 2010 (i.e., through carriers or patients from endemic regions) $(8,30)$. We have shown that over a 30-year span, several local lineages are present at relatively constant levels (Fig. 1C). This underlines that local and pandemic lineages exhibit different epidemiological behaviors, and may occupy different ecological niches in Latin America.

We show that there are local foci of diverse $V$. cholerae lineages that cause sporadic outbreaks across Latin America. Local lineages share many characteristics with pandemic clones, such as being toxigenic and of serogroup O1 (table S3). Disease caused by these lineages would thus be defined as cholera by both the World Health Organization (38) and U.S. Centers for Disease Control and Prevention (39). However, these local lineages show markedly different patterns of dis- ease to that of the 7PET pandemic V. cholerae lineage. The potential of a V. cholerae isolate to cause disease is best understood by studying its genomics, whether by whole-genome sequencing or a polymerase chain reaction-based typing scheme, as well as considering clinical symptoms, epidemiological context, and basic pheno- and serotyping data.

In this study, we have unified previous accounts of cholera within Latin America into a cohesive genomic framework that correctly emphasizes the relative contributions of different bacterial lineages to this diarrheal disease. An appreciation of the differences between pandemic and local lineages should inform the design of disease control strategies in Latin America. Measured and graded public health responses could be designed based on an understanding of which lineages are responsible for outbreaks of cholera. V. cholerae lineages can be prioritized as public health concerns if they deviate from patterns associated with local lineages.

\section{REFERENCES AND NOTES}

1. World Health Organization, Cholera; www.who.int/ mediacentre/factsheets/fs107/en/.

2. D. Ceccarelli, R. R. Colwell, Front. Microbiol. 5, 256 (2014).

3. R. R. Colwell, Science 274, 2025-2031 (1996).

4. E. K. Lipp, A. Huq, R. R. Colwell, Clin. Microbiol. Rev. 15 757-770 (2002)

5. E. T. Ryan, PLOS Negl. Trop. Dis. 5, e1003 (2011).

6. P. A. Blake et al., N. Engl. J. Med. 302, 305-309 (1980)

7. J. Kumate, J. Sepúlveda, G. Gutiérrez, Bull. Inst. Pasteur 96 217-226 (1998)

8. L. S. Katz et al., MBio 4, e00398-13 (2013).

9. Pan American Health Organization/World Health Organization, Cholera in the Americas - Situation summary. Epidemiol. Update Cholera (2017); www.paho.org/hq/index.php?option=com_ docman\& task $=$ doc download\&ltemid=270\&gid=38251\&lang=fr.

10. D. N. Cameron, F. M. Khambaty, I. K. Wachsmuth, R. V. Tauxe, T. J. Barrett, J. Clin. Microbiol. 32, 1685-1690 (1994).

11. M. L. Lizárraga-Partida, M.-L. Quilici, J. Clin. Microbiol. 47 , 1364-1371 (2009)

12. T. Popovic, C. Bopp, O. Olsvik, K. Wachsmuth, J. Clin. Microbiol. 31, 2474-2482 (1993)

13. G. M. Evins et al., J. Infect. Dis. 172, 173-179 (1995).

14. A. Dalsgaard et al., J. Clin. Microbiol. 35, 1151-1156 (1997).

15. Materials and methods are available as supplementary materials.

16. F.-X. Weill et al., Science 358, 785-789 (2017)

17. J. B. Kaper, H. B. Bradford, N. C. Roberts, S. Falkow, J. Clin. Microbiol. 16, 129-134 (1982)

18. M. Pichel et al., J. Clin. Microbiol. 41, 124-134 (2003).

19. A. Coelho, J. R. Andrade, A. C. Vicente, C. A. Salles, J. Clin. Microbiol. 33, 114-118 (1995)

20. M. Alam et al., J. Clin. Microbiol. 48, 3666-3674 (2010).

21. M. Alam et al., J. Clin. Microbiol. 50, 2212-2216 (2012).

22. A. Siriphap et al., PLOS ONE 12, e0169324 (2017).

23. P. A. Blake et al., Lancet 2, 912 (1983).

24. P. Beltrán et al., J. Clin. Microbiol. 37, 581-590 (1999).
25. I. K. Wachsmuth et al., J. Infect. Dis. 167, 621-626 (1993). 26. A. Mutreja et al., Nature 477, 462-465 (2011)

27. M. Alam et al., Proc. Natl. Acad. Sci. U.S.A. 111 9917-9922 (2014)

28. J. P. Guthmann, J. Trop. Med. Hyg. 98, 419-427 (1995).

29. C.-S. Chin et al., N. Engl. J. Med. 364, 33-42 (2011).

30. R. S. Hendriksen et al., MBio 2, e00157-e11 (2011).

31. J. W. Tappero, R. V. Tauxe, Emerg. Infect. Dis. 17, 2087-2093 (2011).

32. S. M. Moore, K. L. Shannon, C. E. Zelaya, A. S. Azman, J. Lessler, PLOS Curr. 6, ecurrents.outbreaks. c04478c7fbd9854ef6ba923cc81eb799 (2014)

33. J. A. Díaz-Quiñonez et al., Genome Announc. 2, e01123-14 (2014)

34. G. H. Rabbani, W. B. Greenough 3rd, J. Diarrhoeal Dis. Res. 17 1-9 (1999)

35. J. Martinez-Urtaza, J. Trinanes, N. Gonzalez-Escalona, C. Baker-Austin, Nat. Microbiol. 1, 16018 (2016).

36. C. Seas et al., Am. J. Trop. Med. Hyg. 62, 513-517 (2000).

37. S. Y. Choi et al., MBio 7, e02160-e15 (2016).

38. World Health Organzation, Prevention and control of cholera outbreaks: WHO policy and recommendations; www.who.int/ cholera/technical/prevention/control/en/index1.html.

39. Centers for Disease Control and Prevention, Cholera (Vibrio cholerae 01/0139). 1996 Case Definition; https://wwwn.cdc. gov/nndss/conditions/cholera/case-definition/1996/.

\section{ACKNOWLEDGMENTS}

This study was supported by the Wellcome Trust (grant 098051), the Institut Pasteur, Santé Publique France, and the French government's Investissement d'Avenir program, Laboratoire d'Excellence "Integrative Biology of Emerging Infectious Diseases" (grant no. ANR-10-LABX-62(BEID). We thank J. Rauzier for technical assistance; A. J. Page, J. Keane, and the sequencing teams at the Wellcome Trust Sanger Institute and the Institut Pasteur; and M. Beale for helpful comments. M.J.D. is supported by a Wellcome Trust Sanger Institute Ph.D. Studentship. A.E.M. is supported by Biotechnology and Biological Sciences Research Council fellowship BB/M014088/1. D.M.A. is supported by the Centre for Genomic Pathogen Surveillance. M.-L.Q. is a member of the WHO Global Task Force on Cholera Control and leader of the Laboratory Surveillance Working Group, involved in developing and disseminating technical guidance. J.P. is a member of the Institut Pasteur Scientific Council and a paid consultant to Specific Technologies. The findings and conclusions in this report are those of the author(s) and do not necessarily represent the official position of the Centers for Disease Control and Prevention. All data and code necessary to understand and assess the conclusions of this research are available in the main text, supplementary materials, and via the following repositories: European Nucleotide Archive (ENA) (www.ebi.ac.uk/ena), under study accession numbers PRJEB9140, PRJEB2215, and PRJEB8764; Figshare https://figshare.com/articles/Integrated_view_of_Vibrio_ cholerae_in_the_Americas/5427253. Phylogeny and metadata can be viewed interactively at https://microreact.org/project/ globalcholera?tt=rc.

\section{SUPPLEMENTARY MATERIALS}

www.sciencemag.org/content/358/6364/789/suppl/DC1 Materials and Methods

Supplementary Text

Tables S1 to S4

Figs. S1 to S11

References (40-86)

27 June 2017; accepted 10 October 2017

10.1126/science.aao2136 


\section{Science}

\section{Integrated view of Vibrio cholerae in the Americas}

Daryl Domman, Marie-Laure Quilici, Matthew J. Dorman, Elisabeth Njamkepo, Ankur Mutreja, Alison E. Mather, Gabriella Delgado, Rosario Morales-Espinosa, Patrick A. D. Grimont, Marcial Leonardo Lizárraga-Partida, Christiane Bouchier, David M. Aanensen, Pablo Kuri-Morales, Cheryl L. Tarr, Gordon Dougan, Julian Parkhill, Josefina Campos, Alejandro Cravioto, François-Xavier Weill and Nicholas R. Thomson

Science 358 (6364), 789-793.

DOI: $10.1126 /$ science.aao2136

\section{Wave upon wave of disease}

The cholera pathogen, Vibrio cholerae, is considered to be ubiquitous in water systems, making the design of eradication measures apparently fruitless. Nevertheless, local and global Vibrio populations remain distinct. Now, Weil et al. and Domman et al. show that a surprising diversity between continents has been established. Latin America and Africa bear different variants of cholera toxin with different transmission dynamics and ecological niches. The data are not consistent with the establishment of long-term reservoirs of pandemic cholera or with a relationship to climate events. Science, this issue p. 785 , p. 789

ARTICLE TOOLS

SUPPLEMENTARY

MATERIALS

RELATED

CONTENT

REFERENCES

PERMISSIONS http://science.sciencemag.org/content/358/6364/789

http://science.sciencemag.org/content/suppl/2017/11/09/358.6364.789.DC1

http://science.sciencemag.org/content/sci/358/6364/706.full http://science.sciencemag.org/content/sci/358/6364/785.full http://stm.sciencemag.org/content/scitransmed/5/192/192ra86.full

This article cites 78 articles, 26 of which you can access for free http://science.sciencemag.org/content/358/6364/789\#BIBL

http://www.sciencemag.org/help/reprints-and-permissions

Use of this article is subject to the Terms of Service

Science (print ISSN 0036-8075; online ISSN 1095-9203) is published by the American Association for the Advancement of Science, 1200 New York Avenue NW, Washington, DC 20005. 2017 (C) The Authors, some rights reserved; exclusive licensee American Association for the Advancement of Science. No claim to original U.S. Government Works. The title Science is a registered trademark of AAAS. 